\title{
Immunmodulatory and Antiproliferative Properties of Rhodiola Species*
}

Authors

Affiliation
Mari-Carmen Recio, Rosa-María Giner, Salvador Máñez

Department of Pharmacology, Faculty of Pharmacy, University of Valencia, Valencia, Spain

\author{
Key words \\ - Rhodiola \\ - salidroside \\ - adaptatogen \\ - antitumor \\ - immunostimulant \\ - antioxidant
}

\begin{abstract}
$\nabla$

The traditional medicines of Asia and Europe have long used various Rhodiola species, which are endemic to the subarctic areas of the northern hemisphere, as tonic, adaptogen, antidepressant, and anti-inflammatory drugs. In order to establish the therapeutic uses of these plants in modern medicine, the pharmacological effects of Rhodiola sp. have been widely studied. Indeed, the most amply researched species, Rhodiola rosea, has been shown to possess antioxidant, adaptogenic, antistress, antimicrobial, immunomodulatory, angiomodulatory, and antitumoral effects. Salidroside ( $p$-hydroxyphenethyl- $\beta$-D-glucoside), a major compound in Rhodiola, seems to be re-
\end{abstract}

\section{Introduction}

$\nabla$

Rhodiola species are herbaceous perennial plants of the Crassulaceae family that have been used extensively in the traditional medicines of both Asia and Europe as tonic, adaptogen, antidepressant, and anti-inflammatory drugs. Rhodiola species grow in cooler regions in the subarctic areas of the Northern hemisphere, including Northern and Central Europe, Asia, and North America. It is believed that the genus Rhodiola originates from the mountainous regions of Southwest China and the Himalayas [1]. Although the current taxonomic status of the genus Rhodiola is quite complex due to a generally similar morphology, a total of 136 species have been identified according to GBIF [2]. From a chemotaxonomical point of view, eight compounds (the phenylpropanoids rosarin, rosavin, and rosin, the phenylethanoids salidroside and tyrosol, the flavonol rhodionin, as well

\footnotetext{
* Dedicated to Professor Dr. Dr. h.c. mult. Kurt Hostettmann in recognition of his outstanding contribution to natural product research.
}

sponsible for many of the effects observed with Rhodiola extracts.

The aim of this paper is to review the pharmacological effects not only of various Rhodiola species, mainly $R$. rosea along with Rhodiola imbricata, Rhodiola algida, and Rhodiola crenulata, but also of salidroside, focusing especially on its antioxidant, immunomodulatory, antitumoral, and antiproliferative activities, as well as to describe their therapeutic significance in disease management. Although previous pharmacological studies have established a scientific basis for possible therapeutic uses of Rhodiola extracts and salidroside, high-quality, randomized, controlled clinical trials are still needed. sea L. However, subsequent studies have shown that in addition to salidroside, rosin derivatives are also important bioactive compounds. In fact, the activity of $R$. rosea extracts was found to be superior to that of the individual compounds, indicating that the aforementioned glycosides are probably not the only compounds responsible for the adaptogenic and immunostimulant properties of the plant. It is worth noting that the $R$. rosea extracts used in most pharmacological studies were standardized to contain a minimum of $3 \%$ rosin and its derivatives and $0.8-1 \%$ salidroside in order to mimic the naturally occurring ratio of these compounds in the $R$. rosea root, which is approximately $3: 1$. 


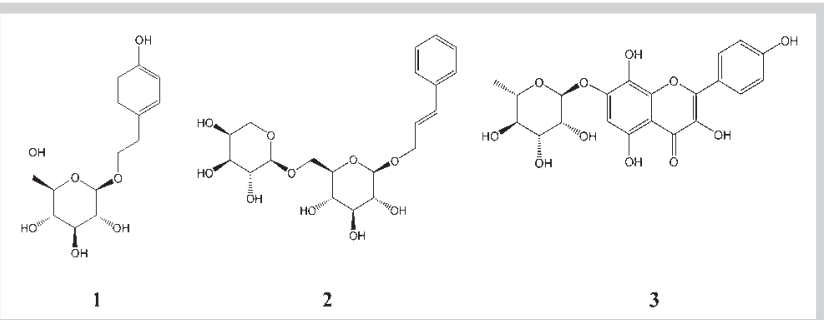

Fig. 1 Chemical structures of salidroside (1), rosavin (2), and rhodionin (3).

The demand for Rhodiola-based products has increased in the past few years, necessitating a greater control over the quality of the raw material supply and its collection from natural sources. Studies have demonstrated that the content of salidroside as well as that of rosin and its derivatives is higher in wild plants than in samples obtained from field crops. Unfortunately, due to the intensive collection of $R$. rosea, the plant is now listed as an endangered species in many countries.

$R$. rosea is the most widely researched species of the genus, with studies demonstrating antioxidant, adaptogenic, antistress, antimicrobial, immunomodulatory, and angiomodulatory properties of the extracts [3]. However, other Rhodiola species [4,5] ( Table 1) have also been studied, including Rhodiola imbricata Edgew, Rhodiola crenulata (Hook.f. \& Thomson) H. Ohba, and Rhodiola kirilowii (Praeger) H. Jacobsen. The latter species is a traditional Chinese herbal drug for the treatment of altitude sickness, and is commonly used by mountaineers, aviators, and astronauts. Rhodiola quadrifida Fisch. \& C.A.Mey. is used in traditional Mongolian medicine, but has been poorly investigated from a phytochemical and pharmacological perspective [6].

In 2010, Panossian et al. [7] published a systematic review of clinical trials to evaluate the level of scientific evidence for the efficacy of $R$. rosea in the treatment of specific conditions (fatigue, depression). More recently, a book was published with the entire body of knowledge and all the ongoing research on $R$. rosea to date, with a substantial number of references from 2010 to 2013, as well as a few from 2014 [8].

In addition, the modulatory effects of Rhodiola spp. and other natural antioxidants on angiogenesis have also been recently reviewed [6]. Apart from their adaptogenic properties, it seems that Rhodiola species also have antitumoral and antiproliferative effects, protecting tissues against free radicals and weak and medium-strength mutagens. One important property of Rhodiola extracts is their ability to inhibit angiogenesis. Even though different Rhodiola species were used, and methods of preparing the extracts differed somewhat in the published studies, the extracts stimulated specific and nonspecific immunity both in vivo and in vitro. Extracts also inhibited skin neovascularization induced in mice through the implantation of syngeneic tumor cells (except for an aqueous extract of $R$. kirilowii) or human kidney cancer homogenate. A similar inhibitory effect was observed in mice fed salidroside and rosavin for 3 days after implantation of tumor cells. In in vitro experiments conducted in cell cultures of mouse endothelial cells in the presence of L-1 sarcoma, the hydroalcoholic extract of $R$. kirilowii inhibited migration and increased proliferation of endothelial cells. Some data suggest that antioxidants affect physiological angiogenesis in vivo through the regulation of nitric oxide synthase (NOS) expression and activity.
Table 1 Distribution of Rhodiola sp. in relevant Holarctic areas [4,5].

\begin{tabular}{|c|c|}
\hline China & 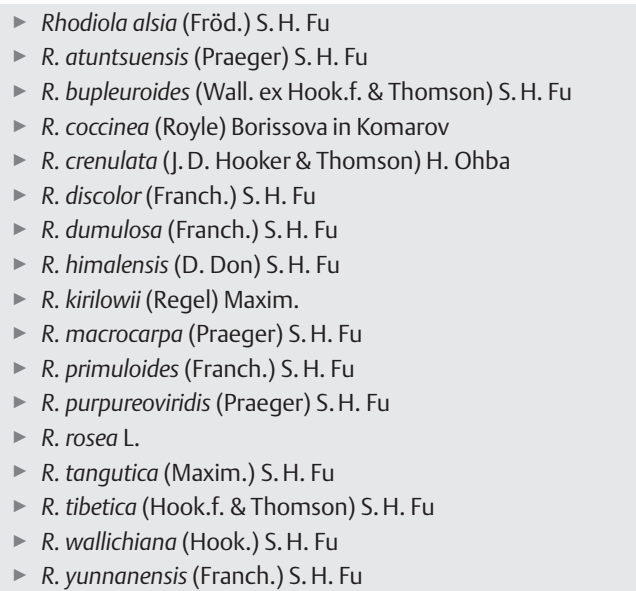 \\
\hline Pakistan & $\begin{array}{l}\text { R. pachyclados (Aitch. \& Hemsl.) H. Ohba } \\
\text { - R. saxifragoides (Fröd.) H. Ohba } \\
\text { - R. recticaulis Borissova in Komarov } \\
\text { - R. heterodonta (Hook.f. \& Thomson) Borissova in Komarov } \\
\text { - R. sinuata (Royle ex Edgew.) S. H. Fu } \\
\text { - R. fastigiata (Hook.f. \& Thomson) S.H. Fu } \\
\text { - R. imbricata Edgew }\end{array}$ \\
\hline Europe & $\begin{array}{l}\text { R. quadrifida (Pall.) Fisch. \& C. A. Mey. } \\
\text { R. rosea L. }\end{array}$ \\
\hline $\begin{array}{l}\text { North } \\
\text { America }\end{array}$ & $\begin{array}{l}\text { R. integrifolia Raf. } \\
\text { - R. rhodantha (A. Gray) H. Jacobsen } \\
\text { - R. rosea L. }\end{array}$ \\
\hline
\end{tabular}

However, the molecular mechanisms involved in these different effects remain unclear, making more studies necessary, both in vitro and especially in vivo.

Panossian et al. [9] recently published a study on the modulation of gene expression in glial cells by treatment with $R$. rosea extract and three isolated compounds. They used the T98G human neuroglial cell line to obtain total RNA, which was transcribed to cDNA, and then submitted to a gene expression profiling procedure comprising 561 genes for extract-treated cells, and 640, 601, and 562 genes for salidroside-, traindrin-, and tyrosol-treated cells, respectively. The authors concluded that Rhodiola extract mainly affects genes associated with cellular development and cell-cell signaling, while tyrosol affects genes associated with molecular transport, nervous system development, and cancer. For its part, triandrin affects genes that play a role in molecular transport and inflammation. In a later study, these same authors published refined data on the same subject, but only for $R$. rosea extracts and principles.

In this paper, we discuss the most recent findings on the pharmacological effects of Rhodiola species and one of its pure bioactive compounds, salidroside, focusing on their antioxidant, immunomodulatory, antiproliferative, and antitumoral activities.

\section{Pharmacological Activities of Rhodiola extracts $\nabla$}

The ability of $R$. rosea to promote the host's immune response has been documented in numerous in vitro and animal studies as well as in a few clinical trials, all of which indicated that $R$. rosea is able to modulate the immune response. The innate immune cells include dendritic cells, macrophages, and neutrophils, among others, while B and T lymphocytes constitute the adaptive 
immune cells. The latter coordinate the immune response and play a key role in cell-mediated immunity. T lymphocyte subsets include helper $\left(\mathrm{CD}^{+}\right) \mathrm{T}$ (Th) cells and suppressor/cytotoxic (CD8 $\left.{ }^{+}\right) \mathrm{T}$ cells, with $\mathrm{CD} 4^{+} / \mathrm{CD}^{+}$being the critical indicator of immune homeostasis. Th1 cells produce inflammatory cytokines such as IL-2, IL-12, IFN- $\gamma$, GM-CSF, and TNF, which induce cell-mediated immune responses, while Th2 cells secrete cytokines such as IL-4, IL-5, IL-6, and IL-10 to induce humoral or allergic responses. In addition, other Th subsets such as Th17 and regulatory T (reg) cells have a presumed role in autoimmune tissue pathology. Cytokines produced by Th subsets play a critical role in immune cell differentiation, effector subset commitment, and in directing the immune system towards suitable responses [10].

One type of analysis for innate immune activation concerns granulocyte activation, measured with various techniques. One of the classic methods for this type of analysis, namely chemiluminescence, was used by Zdanowski et al. [11] to examine the effect of the polar extracts of $R$. rosea on the blood cells of BALB/c mice. The effects were small, except for the observed increase in luminescence after one week of treatment with $0.4 \mathrm{mg}$ of hydroalcoholic extract, which, although low, was the highest dose used in the assay. Surprisingly, this increase was not associated with a higher granulocyte count. Moreover, the aqueous extract, which contained more gallic acid and less rosavin (arabinoglucosyl cinnamate) than the hydroalcoholic extract, actually reduced the number of blood granulocytes. A careful reading of this study is recommended for the purpose of interpreting these differences. The effects of a standardized $R$. rosea extract on cytokine modulation were also studied in an ovalbumin-primed mouse model. Both Th1 (IL-2 and IFN- $\gamma$ and Th2 (IL-4 and IL-10) cytokine levels were significantly higher after daily treatment with the extract (doses up to $200 \mathrm{mg} / \mathrm{kg}$ body weight were shown to be safe at least over a 28-day period) in a dose- and time-dependent manner. Quantitative HPLC analysis determined the content of salidroside in the extract to be $4.39 \%(\mathrm{w} / \mathrm{v})$. However, treatment with salidroside alone was less effective than with the extract, suggesting a synergistic effect with other compounds [12].

Immunosuppression is involved in sepsis, one of the leading causes of mortality in critically ill patients. The extensive apoptosis of B and T lymphocytes and dendritic cells associated with severe sepsis impairs the immune function of surviving cells and compromises the ability of the patient to eradicate the pathogenic infection, resulting in multiple organ failure and mortality [13]. In this process, the tumor necrosis factor- $\alpha$-inducible protein 8-like 2 (TIPE2) is preferentially expressed in lymphoid tissues, negatively regulating the innate and adaptive immune response and maintaining immune homeostasis. In septic mice, $R$. rosea both suppressed T lymphocyte apoptosis and promoted host immunity by downregulating TIPE2. Intraperitoneal injection of an ethanolic extract of the $R$. rosea root $(50 \mathrm{mg} / \mathrm{kg}, 8 \mathrm{~h}$ prior to surgery) protected mice against cecal ligation and punctureinduced sepsis by attenuating the induced changes. Pretreatment with Rhodiola extract increased the survival of septic mice, inhibiting the upregulated expression of TIPE2 and the apoptosis-promoting proteins Fas and FasL. It also increased the downregulated expression of the apoptosis-inhibiting protein $\mathrm{Bcl}-2$, decreased enhanced $\mathrm{T}$ lymphocyte apoptosis, and increased the lowered number of thymus $\mathrm{T}$ lymphocytes, along with $\mathrm{CD}^{+}$, $\mathrm{CD}^{+}$, and $\mathrm{CD}^{+} / \mathrm{CD}^{+}$subsets [14]. Human sepsis involves an early proinflammatory phase followed by a late compensatory anti-inflammatory period, with a beneficial role for an increased number of Th1 cytokines in the latter stage when immunosup- pression predominates. Although this experimental sepsis induced both Th1 and Th2 cytokine production, $R$. rosea extract specifically enhanced the Th1 cytokines IFN- $\gamma$, IL-2, and IL-12, but did not affect Th2 cytokine levels, thus improving immunity [14].

The immunostimulant potential of $R$. rosea was determined in a 45 -day head-down bed rest model at $-6^{\circ} \mathrm{C}$, the most frequently used method for evaluating multisystem responses to microgravity in humans during spaceflight. In this Chinese study, 15 healthy male volunteers were randomly selected and placed into either the placebo $(n=8)$ or the $R$. rosea treatment group $(n=7)$. The latter group received $1.0 \mathrm{~g}$ of $R$. rosea per day from day 1 to day 7 as a prophylactic dose, followed by $2.0 \mathrm{~g}$ per day from days 8 to 45 as a therapeutic dose during head-down bed rest. Deregulation of the immune system, evaluated in peripheral blood samples at different times throughout the study, included changes in cell subsets (increased percentages of memory $\mathrm{T}$ and $\mathrm{B}$ cells, Tregs, and monocytes), alterations in cytokine production patterns (increases in IL-1 $\beta$ and IL-18, and decreases in IFN- $\gamma$ and IL-17 production), as well as changes in antibody production (increased IgE). Moreover, treatment with $R$. rosea suppressed proinflammatory cytokines, decreasing both IFN- $\gamma$ production by $\mathrm{T}$ cells and upregulation of the IL-1 family of cytokines by various blood cell types, but did not attenuate the T cell immune response [15]. $R$. rosea and $R$. quadrifida extracts exerted a dose-dependent effect on the proliferative response of mouse splenic lymphocytes to another T cell mitogen, namely Phaseolus vulgaris hemagglutinin, with response stimulation when mice were fed with lower doses and inhibition of proliferation at higher doses [16].

Among the natural sources of hypopigmentation agents, phenolic compounds of plant origin appear to be the most effective. Most studies on hypopigmentation have focused on the synthesis and activity of melanin and tyrosinase, the rate-limiting enzyme in melanogenesis. Chiang et al. [17] found that an $R$. rosea hydroalcoholic extract and hydrolysate inhibited melanin synthesis and tyrosinase activity in B16F0 melanoma cells. The antioxidant effect of $R$. rosea, which decreases radical oxygen species (ROS) production, affected the oxidation of DOPA to DOPA-chrome by suppressing cellular tyrosinase activity, indicating that $R$. rosea inhibits melanin synthesis. Treatment of B16F0 cells with either a hydroalcoholic extract or its hydrolysate led to a significant inhibition of melanocortin 1 receptor (MC1R) expression, which in turn suppressed the expression of downstream melanogenic proteins such as tyrosinase, along with the major transcription activator of tyrosinase expression, namely microphthalmia-associated transcription factor. Treatment with $R$. rosea $(200 \mu \mathrm{g} / \mathrm{mL})$ suppressed the expression of tyrosinase by inhibiting the activation of c-AMP response element binding protein (CREB), and increasing the levels of AKT (protein kinase B) and glycogen synthase kinase-3 $\beta$ (GSK3 $\beta$ ) expression in melanoma cells. Further investigations of $R$. rosea on normal melanocytes, including in vivo studies, are needed to corroborate these findings.

A hydroethanolic extract of $R$. rosea rhizome [18] protected human brain cortical cells ( $\mathrm{HCN} 1-\mathrm{A}$ ) against $\mathrm{H}_{2} \mathrm{O}_{2}$ and glutamate toxicity. Thus, at concentrations ranging from $0.1-100 \mu \mathrm{g} / \mathrm{mL}$, the extract enhanced cell viability and protected normal polarized morphogenesis in vitro. Treatment with $\mathrm{H}_{2} \mathrm{O}_{2}$ augmented the intracellular $\mathrm{Ca}^{2+}$ concentration from $132 \mathrm{nM}$ to $310 \mathrm{nM}$, whereas in the presence of $100 \mu \mathrm{g} / \mathrm{mL}$ of extract, the concentration reached nearly $220 \mathrm{nM}$. Interestingly, a cooperative behavior was observed with verapamil, a known calcium channel blocker, in terms of restoring $\mathrm{Ca}^{2+}$ concentration altered by glutamate. 
Several studies have demonstrated the antitumoral activities of $R$. rosea extracts. For instance, the commercial extract SHR-5 selectively inhibited the growth of bladder cancer cell lines and p53 defective cells with a minimal effect on nonmalignant bladder epithelial cells. The mode of action of the $R$. rosea extract was related to the inhibition of the mTOR pathway as well as to translation initiation and induction of autophagy via activation of AMPK- $\alpha$ in bladder cancer UMUC-3 cells. A major role of AMP kinase is to act as an energy (AMP/ATP ratio) sensor to inhibit energy consuming processes, including cellular proliferation, under energy deprivation conditions in order to maximize the chance of survival. The authors suggested that the decrease in ROS production brought on by $R$. rosea extracts was due to their effect on oxygen consumption, which leads to a change in ATP production and uncoupling in the mitochondria [19].

Cai et al. [20] conducted in vitro and in vivo studies to examine the inhibitory effect on tumor growth of a polysaccharide fraction isolated from $R$. rosea, which turned out to be a homogeneous heteroglycan. The proliferation of sarcoma 180 cells treated with various concentrations $(25,50$, and $100 \mu \mathrm{g} / \mathrm{mL})$ of the compound for 24,48 , and $72 \mathrm{~h}$ was inhibited in a concentration-dependent manner, mainly after $48 \mathrm{~h}$. The antitumoral activity of the polysaccharide in vivo was determined in a system using sarcoma 180 cells that were implanted in mice by i.p. injection to form solid tumors. Daily administration of polysaccharide $(25,50$, or $100 \mathrm{mg} / \mathrm{kg}$, i.p.) for 10 days resulted in a dose-dependent reduction of tumor growth. A flow cytometry study of the peripheral blood $\mathrm{T}$ lymphocyte cells in tumor-bearing mice showed a high ratio of CD4+/CD8+ T lymphocytes. The polysaccharide had a direct cytotoxic effect in vitro and enhanced the immune response, promoting secretion of IL-2, TNF- $\alpha$, and IFN- $\gamma$. $R$. imbricata is a perennial herb, commonly known as golden or arctic root, which grows in dry areas of the western Himalayas. It is the major constituent of an herbal antioxidant tea developed and patented as a medical supplement in India.

An aqueous extract of $R$. imbricata rhizome was found to stimulate Th1 cytokines (IL- $1 \beta$, IL-6, and TNF- $\alpha$ ) in human peripheral blood monocytes. Treated splenocytes exhibited increased tolllike receptor-4 (TLR-4) expression and intracellular granzyme-B production, which is associated with increased natural killer or cytotoxic cell activity. TLR-4 activation led to an increased expression of NF- $k$ B and may be responsible for the increased activity of Th1 cytokines [21]. The immunomodulatory effect of $R$. imbricata was also evaluated in both a human T cell lymphoma cell line (EL-4) and an erythroleukemic cell line (HL-60), with administration of the compound causing a significant inhibition of their proliferation. Treated human monocytes exhibited increased TNF- $\alpha$ levels, crucial for host survival after infection, while LPS-induced RANTES production was suppressed [22].

Innate immunity is pivotal in the body's defense against viruses, especially because genomic variations of the pathogen are often quicker than cellular and humoral adaptation. Currently, there is growing interest in finding ways to treat viruses transmitted by insects, such as dengue viruses [21]. In this context, Diwaker et al. [23] infected peripheral human blood and THP-1 line monocytes with the RNA of this virus in order to determine the expression of several proteins related to cellular response, including toll-like receptors, IFN $\beta$, the RNA helicase melanoma differentiation-associated protein 5 (MDA5), and retinoic acid-inducible gene I (RIG-I). Cells were cultured during $48 \mathrm{~h}$, after which an aqueous extract of the rhizome of $R$. imbricata $(50 \mu \mathrm{g} / \mathrm{mL})$ was administered $2 \mathrm{~h}$ after the infection, leading to an increase in the expression of the four proteins. In addition, in what appeared to be an orchestrated response, the extract not only reduced viral cell load, but also increased the expression of NF- $k \mathrm{~B}$, cytokines TNF- $\alpha$ and IL- $1 \beta$, and the natural killer lymphocyte surface marker.

Acetone and methanol extracts of $R$. imbricata showed comparable antioxidant activity to that of butylated hydroxyl toluene and rutin [24]. They also inhibited the proliferation of an HT-29 human colon cancer cell line upon treatment at higher concentrations $(200 \mu \mathrm{g} / \mathrm{mL})$. The acetone extract inhibited proliferation in a concentration-dependent manner, whereas the methanol extract showed both concentration- and time-dependent inhibitory activity [24].

Several papers have reported on the ability of Rhodiola extracts or products to alleviate the damage caused by radiation. In one study, the antioxidant properties of the polar extracts of the plant were assessed in an in vitro model based on the ${ }^{60} \mathrm{Co}$-irradiation of linoleic acid in the presence of various copper and iron cations [25].

$R$. crenulata has long been used in traditional Eastern medicine as an adaptogen and antidepressant, and has been administered in doses of up to $680 \mathrm{mg} /$ day with no adverse effects. $R$. crenulata extracts have been shown to have beneficial properties including anti-hypoxia, increased endurance, and antineoplastic effects. In fact, several studies have demonstrated the therapeutic potential of $R$. crenulata in a variety of neoplasias, including breast cancer, bladder cancer, and glioblastoma [26].

One of the most important cytokines in regulating macrophage and Th lymphocyte-driven immunological processes is IFN- $\gamma$. Levels of this cytokine were found to be increased in vitro by different phenolic compounds obtained from $R$. crenulata. In order to study this effect, cultured spleen cells obtained from BALB/C mice were stimulated with concanavalin $A$. The most active compounds were two pseudolignans, two flavonols rhamnoglucosides, and feruloyl- $\omega$-hydroxy-methyl- $n$-hexanoate [26].

The enhancement of low-specificity immune functions was studied by evaluating the response of the intestinal system of the fly Drosophila melanogaster to an extract of $R$. crenulata [27]. The bacteria Serratia marcescens and Micrococcus luteus, along with spores of the fungus Beauveria bassiana, were all used to stimulate tissue alterations, pro-oxidative responses, and gene expression of antimicrobial peptides. Treatment with the extract prolonged the life span of the flies, ameliorated morphological changes in the mucosa, made melanotic masses disappear, and increased the expression of defensive peptides.

Breast cancer is the most common type of cancer among women, with over $70 \%$ of all diagnosed cancer cases testing positive for estrogen receptors (ER). Typically, when this receptor is upregulated, cells are more responsive to endogenous estrogen in the body. In their work, Bassa et al. [28] found that hydroalcoholic extracts of $R$. crenulata not only contain estrogenic components, but also affect ER gene expression in normal mammary epithelium in vivo. Continuous treatment with the extract decreased MCF7 cell proliferation, ER expression levels, and tumorsphere formation. Their results suggested that reduced transcriptional activity of $\beta$-catenin and the ER response were both implicated. Similar results were reported by Mora et al. [29]. Treatment of human U87 glioblastoma (GBM) cells with $200 \mu \mathrm{g} / \mathrm{mL}$ of hydroalcoholic extract of $R$. crenulata suppressed cell proliferation, stimulated differentiation, and eliminated tumorsphere formation of GBM cells in vitro. The inhibitory effects seemed to be related to the inhibition of the $\mathrm{Wnt} / \beta$-catenin signaling pathway. 
In another study, a hydroethanolic extract of $R$. crenulata was tested in a melanoma cell line [30]. B16-F10 murine melanoma cells were treated with $200 \mu \mathrm{g} / \mathrm{mL}$ of extract for 24,48 , and $72 \mathrm{~h}$. The extract induced morphological changes and decreased the proliferation and survival of cells in a time-dependent manner. In addition, inhibition of B16-F10 melanoma cell migration was observed at doses of $100 \mu \mathrm{g} / \mathrm{mL}$ over a 24 -h period. To evaluate the antitumoral effect in vivo, experiments using a subcutaneous syngenic melanoma tumor model in C57BL/6 mice were performed. Tumors were treated topically with $R$. crenulata extract in a Eucerin ${ }^{\mathrm{TM}}$-based cream. An interesting difference was observed in the pattern of tumor growth between treatment groups, with a definite trend toward increased survival in the mice treated with $R$. crenulata. In addition, the number of established tumor foci in the lungs of treated mice was notably reduced compared to the control group. Considering these findings, it seems that $R$. crenulata may have potential as an adjuvant in the treatment of melanoma, but obviously more studies are needed.

Rhodiola algida is widely used in traditional Chinese medicine to stimulate the immune system. $R$. algida extract was demonstrated to stimulate human peripheral blood lymphocytes, probably via regulation of IL-2 in Th1 cells, and IL-4, IL-6, and IL-10 in Th2 cells [31]. Loo et al. [32] investigated the effect of an aqueous extract of this plant (at a dose of $100 \mu \mathrm{g} / \mathrm{mL}$ for $48 \mathrm{~h}$ in isolated healthy human lymphocytes in vitro) on both the homeostasis of cancer patients and the healing time of oral ulcers. $R$. algida exhibited no toxicity in animals that had been orally fed with $1 \mathrm{mg} /$ $\mathrm{mL}$ for 30 days. In another study conducted between 2006 and 2007, 130 breast cancer patients were recruited and given four cycles of 5-fluorouracil, epirubicin, and cyclophosphamide after a modified total mastectomy. The patients were randomly assigned to test and control groups. After each cycle of chemotherapy, the treatment group consumed $200 \mathrm{~mL}$ of boiled $R$. algida at a concentration of $50 \mathrm{mg} / \mathrm{mL}$ for 7 consecutive days. The results showed that the optimal concentration assayed in vitro favored not only the proliferation of lymphocytes, but also of IL-2, IL-4, and GMC-SF, along with the mRNA content of these cytokines. Moreover, white blood cell levels returned to normal faster in patients using $R$. algida. These patients also presented smaller and fewer oral ulcers, a common adverse effect of cytotoxic drugs. The results showed that $R$. algida may boost the immune system of both healthy and immunosuppressed patients, and may have potential as an adjuvant treatment to improve the quality of life of such patients.

$R$. algida var. tangutica is a traditional Tibetan herb that has been studied extensively in the past few years. The extract of this plant was shown to inhibit the division of MCF-7 breast cancer cells through a mechanism related to the induction of apoptosis. The transcriptional factor HIF plays an essential role in the adaptive response of cells to reduced oxygen tension. The induction of the transcriptional factor HF1- $\alpha$ is a critical step in the induction of the hypoxic response and occurs via increased mRNA expression, protein stabilization, and nuclear localization. MCF-7 cells cultured under hypoxic conditions were exposed to $R$. algida var. tangutica extract at concentrations of $45-360 \mu \mathrm{g} / \mathrm{mL}$ for $48 \mathrm{~h}$ and showed no signs of toxicity. Cell proliferation under these conditions decreased upon treatment with the plant extract due to induction of apoptosis. Thus, while hypoxia increased the expression of HIF- $1 \alpha$ and HIF- $2 \alpha$ in MCF-7 breast cancer cells, treatment with 225 and $360 \mu \mathrm{g} / \mathrm{mL}$ of $R$. algida var. tangutica prevented the hypoxia-induced proliferation of MCF-7 cells and downregulated the expression of HIF- $1 \alpha$ and HIF- $2 \alpha$ [33].

\section{Pharmacological Data Concerning the Pure Compound Salidroside}

$\nabla$

Salidroside ( $p$-hydroxyphenethyl- $\beta$-D-glucoside) is a major phenylpropanoid glycoside present in Rhodiola species. Various pharmacological properties, including antiaging, neuroprotective, anti-inflammatory, hepatoprotective, and antioxidant effects have all been reported for this compound [34-38]. In addition, studies have demonstrated the antitumoral effects of salidroside through its inhibition of cell proliferation, arrest of the cell cycle, and induction of apoptosis in human bladder, breast, lung, and liver cancer cells $[39,40]$. Salidroside was also shown to inhibit metastasis and angiogenesis $[41,42]$.

Humoral and cell-mediated responses both decrease with aging. The ability of salidroside to improve the body's defense mechanisms and to enhance longevity was studied in a D-galactoseinduced mouse model. Salidroside was found to improve immune function in rats of advanced age after antigen challenge. The compound was able to protect against aging by enhancing age-dependent parameters, such as depressed $\mathrm{T}$ cell function and $\mathrm{T}$ cell-mediated immune response. The rejuvenating activity of salidroside was exerted through an increase in total $\mathrm{T}$ cells $\left(\mathrm{CD}^{+}\right)$and Th cells $\left(\mathrm{CD} 4^{+}\right)$in older rats (21 months old), as well as by increasing the delayed-type hypersensitivity response. It also improved antigen-driven responses by promoting the production of both anti-KLH IgG2 $\alpha$ by Th1 cytokines and anti-KLH IgG1 by Th2 cytokines, all without interfering with immune homeostasis [43].

Certain herbal extracts and pure compounds have been reported to possess adjuvant activity after coadministration with vaccines, which activated innate immunity and stimulated the secretion of cytokines, all with minimal side effects for the host. In this context, various studies have examined whether salidroside can improve the response of elderly subjects to vaccines by enhancing humoral and cell-mediated immune responses. The immunological adjuvant activity of salidroside has been explored as a liposome formulation both in vitro and in vivo, with studies revealing its potential to act as an effective sustained-release vaccine delivery system. To this end, salidroside and the antigen ovalbumin were encapsulated into liposome, a known immune adjuvant and effective drug carrier. Salidroside liposome formulation promoted the stimulation of dendritic cells on mixed leukocyte reaction and improved the antigen presenting ability and maturation of dendritic cells in vitro. The formulation showed a controlled release in vivo, along with prolonged exposure. It enhanced lymphocyte proliferation and serum concentrations of IgG, IL-2, and IFN- $\gamma$, indicating a marked Th1 immunostimulant activity. Thus, salidroside promoted the humoral and cellular immune response while simultaneously regulating the balance of Th1 and Th2 pathways [44].

Salidroside may also be useful in the treatment of autoimmune hepatitis. The efficacy of salidroside in the prevention of immune mediated hepatitis in mice was investigated in a concanavalin A-induced hepatitis model. Intravenous injection of salidroside (50 mg/kg), followed by concanavalin A administration, attenuated induced hepatitis. Salidroside exerted its protective effect against liver injury by reducing plasma alanine transaminase and aspartate transaminase levels, while also ameliorating hepa- 
tocyte necrosis. In addition, it suppressed the secretion of proinflammatory cytokines TNF- $\alpha$, IFN- $\gamma$, and IL-6, partly by downregulating NF- $k$ B phosphorylation. Concomitantly, a notable decrease in $\mathrm{CD}^{+}$and $\mathrm{CD}^{+} \mathrm{T}$ lymphocyte infiltration in the liver was mediated by downregulating CXCL-10 [45].

Salidroside may also regulate bone metabolism via the bone morphogenic protein (BMP) pathway, one of the main signaling cascades involved in osteoblast differentiation and bone formation. Salidroside was shown to stimulate BMP in both pluripotent mesenchymal (C3H10T1/2) and osteoblastic (MC3T3-E1) cell lines, reversing ovariectomy-induced bone loss in rats. This effect is partly due to the promotion of bone formation, since salidroside showed little effect on osteoclast activity [46].

A number of studies have demonstrated a protective effect of Rhodiola species and some of their constituents on various cells and tissues subjected to reactive oxygen species (ROS) (or to conditions that lead to their generation), which constitutes a feature of many inflammatory and degenerative diseases.

In a study with hippocampal cells obtained from Sprague-Dawley rat embryonic brains, salidroside moderately reduced the cell viability loss induced by $30 \mu \mathrm{M} \mathrm{H}_{2} \mathrm{O}_{2}$. When lactate dehydrogenase activity was measured as an indicator of cellular damage, pretreatment with $240 \mu \mathrm{M}$ salidroside lowered the activity to half the control value. Staining with Hoechst 33242 revealed signs of apoptosis, including nuclear shrinkage and chromatin condensation, in $35 \%$ of the cells. Although the number of cells affected was reduced to $15 \%$ by pretreatment with $240 \mu \mathrm{M}$ salidroside, the activity of caspase- 3 was reduced in a lesser manner. However, a slightly higher effect was observed in experiments involving NO release and NOS activity at both 120 and $240 \mu \mathrm{M}$ [47].

Another study made use of PC-12, a class of highly useful cells derived from a rat pheochromocytoma, to evaluate the effects of salidroside on $\mathrm{H}_{2} \mathrm{O}_{2}$-induced apoptosis in neuronal cells. In this experiment, PC-12 cells were stimulated with NGF. Both $\mathrm{H}_{2} \mathrm{O}_{2}$ and salidroside concentrations were lower than those used in the previous study. Thus, treatment with $1 \mu \mathrm{M} \mathrm{H}_{2} \mathrm{O}_{2}$ for $90 \mathrm{~min}$ reduced cellular viability to $49 \%$, whereas pretreatment with $32 \mu \mathrm{M}$ salidroside restored viability to $71 \%$, with concomitant improvements in apoptotic morphological changes. A decrease in caspase-3 activity was also noted, but with a very poor dose relationship. The proposed mechanism of action for salidroside involved the activation of ERK-1/2 phosphorylation [48]. Other authors tested salidroside with the same cells in order to study the apoptosis induced by the experimental neurotoxin 1-methyl-4phenylpyridinium $\left(\mathrm{MPP}^{+}\right)$. Salidroside partly restored nuclear morphology and mitochondrial membrane potential; it also lessened the apoptotic damage, measured in terms of annexin-V expression and propidium iodide staining [49]. Inhibition of NO synthesis by L-NMMA mediated the recovery of cell viability. In addition, salidroside lowered the increase in NO as measured with DAF-FM while also reducing the formation of bound 3nitrotyrosine, inhibiting $\mathrm{Ca}^{2+}$ influx, and decreasing the expression of both iNOS and nNOS. With all these effects, the compound may act as a neuroprotector in degenerative diseases characterized by excitotoxicity mediated by ROS, including NO and its metabolites. In subsequent research, salidroside was reported to enhance phosphorylation of Akt, a Ser/Thr kinase implicated in neuronal cell survival routes [50]. This finding bolsters the hypothesis of the possible role of phenolic glucoside in protecting against the progression of Parkinson's disease.

HUVEC cells were used to analyze the role of micro-RNA (miRNA) in the protective effect of salidroside against oxidative damage
[51]. Exposure of cells to increasing concentrations of $\mathrm{H}_{2} \mathrm{O}_{2}$ produced a decrease in the expression of miR-103, a miRNA that protects against obesity and stroke by regulating the expression of the mitochondrial BCL2/adenovirus E1B interacting protein (BNIP3). Overexpression of miR-103 diminished ROS production and recovered the viability of $\mathrm{H}_{2} \mathrm{O}_{2}$-treated cells. Treatment with salidroside enhanced miR-103 while also inhibiting BNIP3 expression, which is upregulated in several pathological conditions of the ischemic heart.

Another study used cobalt(II) chloride as a hypoxia-mimicking and proapoptotic agent to assess whether salidroside prevented such effects in cultured EA.hy926 endothelial cells [52]. After treatment with $\mathrm{CoCl}_{2}$ for $24 \mathrm{~h}$, nearly $20 \%$ of the cells suffered apoptotic changes; treatment with salidroside at 1 and $10 \mu \mathrm{g} / \mathrm{mL}$ reduced that number to $13 \%$ and $10 \%$, respectively. In a similar fashion and at the same concentrations, salidroside significantly reduced the intracellular accumulation of ROS induced by $\mathrm{CoCl}_{2}$. Western blot analyses demonstrated that salidroside inhibited the upregulation apoptosis markers caspase- 3 and Bax protein, but had less influence on the hypoxia inducible factor (HIF)- $1 \alpha$.

Hu et al. [53] reported for the first time the effect of salidroside on the proliferation of six human cancer cells lines treated with increasing concentrations of the compound (up to $32 \mu \mathrm{g} / \mathrm{mL}$ ) for $48 \mathrm{~h}$. Salidroside inhibited the growth of cancer cells in a concentration- and time-dependent manner, with MDA-MB-231 and A549 being the most sensitive cells ( $\mathrm{IC}_{50}=3.2$ and $4.3 \mu \mathrm{g} / \mathrm{mL}$, respectively). Higher concentrations inhibited all tested cancer cell lines. Analyses of the cell cycle progression showed that salidroside causes $G_{1}$-phase and/or $G_{2}$-phase arrest either by decreasing the activity of the CDK4 cyclinD1 pathway and/or by modulating the Cdc2- Cyclin B1 pathway, respectively. The authors observed that salidroside inhibited the ER negative human breast cancer MDA-MB-231 cells at lower concentrations than hormone sensitive MCF-7 cells, indicating a possible interaction of salidroside with the steroid receptor. The effects of salidroside (up $80 \mu \mathrm{M}$ for $48 \mathrm{~h}$ ) on cell growth characteristics such as proliferation, cell cycle duration, and apoptosis were thus evaluated in both breast cancer cell lines. ER-negative MDA-MB-231 cells were more susceptible to salidroside $\left(\mathrm{IC}_{50}=10 \mu \mathrm{M}\right)$ than ER-positive MCF-7 cells $(20 \mu \mathrm{M})$. Under the conditions of this particular experiment, the $\mathrm{IC}_{50}$ of tamoxifen on MCF-7 cells was $30 \mu \mathrm{M}$, indicating that the effect of salidroside was greater than that of the nonsteroidal estrogen antagonist, which is widely used to treat estrogen-positive breast cancer. It was originally thought that to induce the death of ER-positive MCF-7 cells, salidroside acted as an estrogen antagonist; however, a receptor-binding assay showed that salidroside was not an antagonist at all. In fact, salidroside induced apoptosis of MCF-7 and MDA-MB-231 cells by downregulating the expression of the antiapoptotic proteins $\mathrm{Bcl}-2$ and upregulating the expression of both Bax and cleaved caspase 9 in a concentration-dependent manner.

The effect of purified salidroside on the growth of human breast cancer was studied both in vitro and in vivo [54]. Proliferation of MCF-7 cells incubated with various concentrations of salidroside was significantly inhibited; the compound also induced cell apoptosis and cell cycle arrest at the $G_{0} / G_{1}$ phase in vitro. In addition, salidroside treatment significantly suppressed tumor growth in vivo in a nude mouse model. Further investigation of its possible molecular mechanisms showed that salidroside treatment significantly inhibited intracellular ROS formation and MAPK pathway activation, which may contribute to the reduction of oxidative stress and inhibition of tumor growth in breast cancer. 
Salidroside's anti-metastasis effect in vitro and its mechanisms of action on the MAPK signaling pathway were studied using the human fibrosarcoma HT1080 as a culture cell [55]. Treatment with different concentrations of salidroside (ranging from 10 to $100 \mu \mathrm{mol} / \mathrm{L}$ ) inhibited cellular proliferation slightly at $50 \mu \mathrm{mol} / \mathrm{L}$. Since salidroside showed no obvious morphological changes at this concentration, its anti-metastasis effect was analyzed at 10 , 20 , and $40 \mu \mathrm{mol} / \mathrm{L}$. This study demonstrated for the first time that salidroside inhibited both the migration and invasion of HT1080 cells in a concentration-dependent fashion.

ROS have been identified as important messengers involved in the transduction of several signaling pathways for gene expression and cell proliferation. One of these is the MAPK pathway, which includes ERK, JNK, and p38. Salidroside decreased intracellular ROS formation and the phosphorylation of ERK $1 / 2$ in HT1080 cells, which may be due to an inhibitory effect of salidroside on MAPK activation [55]. Similar results were observed by Wang et al. [56] working with the human alveolar adenocarcinoma cell line A549. Salidroside significantly decreased the invasion index of A549 cells induced by TGF $\beta$, upregulating E-cadherin expression and downregulating $\beta 1$-integrin expression. Salidroside also decreased ROS generation in A549 cells in a doseand time-dependent manner. It is worth noting that pretreatment with antioxidant vitamin $C$ eliminated apoptosis induction by salidroside, indicating that the compound may require a high state of oxidative stress to act. Salidroside significantly decreased the expression of phosphor-p38 protein, a signaling protein associated with oxidative stress. However, in A549 cells pretreated with vitamin $C$, salidroside did not further decrease phosphorp38 protein levels. In addition, salidroside downregulated the expression of Snail, an epithelial-mesenchymal transition marker gene [56].

Glioma, a type of primary central nervous system tumor arising from glial cells, usually occurs in the brain. They are difficult to treat, with many gliomas recurring even after treatment. Zhang et al. [57] proposed salidroside as a potential compound for human tumor treatment. In vitro treatment of human glioma cells U251 with salidroside at $20 \mu \mathrm{g} / \mathrm{mL}$ for $24-72 \mathrm{~h}$ showed cytotoxic effects and growth inhibition, with arrest in the $G_{0} / G_{1}$ phase. In vivo, xenotransplanting the tumor into nude mice induced a sudden decrease in body weight due to the quick growth of the xenotransplanted tumor. However, this decrease was much slower in the salidroside treatment group $(50 \mathrm{mg} / \mathrm{kg} / \mathrm{day}$, i.p. for 20 days) due to a slower tumor growth. Furthermore, the physical and mental status of mice in the salidroside group seemed to be better than in the control group. The authors also found that salidroside inhibited the level of ROS by detecting the 8-isoprostane biomarker of oxidative stress. The downregulation of this biomarker reduces the overgrowth of astrocytes, thus normalizing their formation and growth.

The effect of salidroside on cell proliferation, apoptosis, migration, and invasion was also studied in the colon SW1116 cell line [58]. In addition to its known antitumoral effects, salidroside inhibited the phosphorylation of the JAK2 and STAT3 signaling pathways. STAT3 is activated in many different tumor cells, making it a prime target for treating colon cancer.

Taking all these results into account, the antitumoral effects of salidroside involve a variety of molecules and signaling pathways, including cyclin D1, mTOR, and HIF- $1 \alpha$, as well as the MAPK, VEGF, STAT3, and JAK2 pathways, among others, but the specific mechanisms remain unclear.
A good approach for investigating salidroside's protective effect against radiation was elaborated by Li et al. [59], who assayed three active principles from different adaptogen plants and measured the effects in an in vivo murine model. BALB/c mice were exposed to one dose of $6 \gamma$-rays, after having received the drug intragastrically once daily for one week. Fourteen days after irradiation, the leukocyte count was $4.4 \times 10^{6} / \mathrm{mL}$ in contrast to $12.3 \times 10^{6} / \mathrm{mL}$ for the non-radiated control group. Animals treated with $40 \mathrm{mg} / \mathrm{kg}$ of salidroside had a $7.0 \times 10^{6} / \mathrm{mL}$ count. The radiation also produced changes in the cell cycle of the bone marrow cells, observed mainly as an increase in the ratio of cells in the $G_{0} / G_{1}$ phase (60\% to $87 \%$ ), and a decrease in the ratio of cells in the $\mathrm{G}_{2} / \mathrm{M}$ phase (10\% to $2 \%$ ) in only one day. Pretreatment with salidroside corrected this distribution, increasing the ratio of cells in the $G_{0} / G_{1}$ phase to $65 \%$. Moreover, the compound alleviated other effects of radiation, such as increases in NF- $k$ B, Bax protein, and DNA degradation.

One of the effects of oxidative stress in bone marrow is that hematopoietic stem cells (HSC) are recruited from their quiescent form to the cell cycle. This process should be considered an integral part of homeostasis since redox status is the result of the equilibrium of physiological counterforce. In order to study these processes, Lin-c-kit ${ }^{+} \mathrm{Sca}-\mathbf{1}^{+}$(LSK) cells have often been used as a canonical marker. In vivo treatment of C57BL/6 mice with $\mathrm{H}_{2} \mathrm{O}_{2}$ increased the frequency of LSK cells, but with a lowered fraction of long-term $\mathrm{CD}^{-} 4^{-} \mathrm{Flt3}^{-}$LSK cells. The administration of salidroside $(75 \mathrm{mg} / \mathrm{kg}$ i.p.) not only reduced both alterations, but also facilitated hematopoiesis after irradiation in mice that had received transplanted LSK cells from $\mathrm{H}_{2} \mathrm{O}_{2}$-treated mice. Furthermore, salidroside increased the proportion of quiescent cells (in the $\mathrm{G}_{0}$ phase; negative staining of DNA with pyronin) and decreased cell proliferation in terms of bromodeoxyuridine labeling, generation of 8-oxodeoxyguanosine, and DNA breakage. Many of these effects were found to be unrelated to the inhibition of ROS production or reactivity; instead, salidroside activated poly(ADP-ribose)polymerase-1, a nuclear enzyme able to affix to and repair DNA strand breaks [60].

An interesting approach to the traditional uses of Rhodiola products in respiratory pharmacology is that elaborated by Li et al. [61], who used salidroside to treat HBE16 human airway epithelial cells. The compound helped limit the cellular response to cold stress, established at $18^{\circ} \mathrm{C}$. The main findings point to the transient receptor potential melastatin 8 (TRPM8) channel, which belongs to the once-orphan TRP family of ligand-gated channels, with some degree of selectivity for $\mathrm{Ca}^{2+}$, and opens when the temperature falls below $20^{\circ} \mathrm{C}$. In the context of cold stress, this channel is noteworthy because it enhances the secretion of mucin. Salidroside treatment for $24 \mathrm{~h}$ not only downregulated the expression of mucin $5 \mathrm{~A}$ and TRPM8 protein, but also decreased the intensity of inward $\mathrm{Ca}^{2+}$ current. The mechanism of action for the downregulation of TRPM involved the cAMP response CREB, as seen by the lessening of the effect of salidroside in the presence of CREB siRNA.

Among the different effects of salidroside on various metabolic processes, several authors have examined its usefulness in treating the renal complications of diabetes mellitus. High blood glucose levels cause biphasic effects on mesangial cells, which are key in diabetic nephropathy; first they suffer a proliferative stage, then an arrest in the $\mathrm{G}_{1}$ phase, accompanied by hypertrophy. The authors incubated immortalized human mesangial cells with 1,10 , and $100 \mu \mathrm{M}$ salidroside. Treatment with the highest concentration led to virtual abolition both of cell proliferation caused 
by $25 \mathrm{mM}$ glucose and ROS generation. Moreover, salidroside also reduced the secretion of TGF $\beta$ - 1 and the phosphorylation of ERK 1 and 2 , two events that are ultimately responsible for the proliferative and morphological alterations in this disease [62].

\section{Conclusions}

\section{$\nabla$}

In addition to the known properties of Rhodiola extracts and salidroside, they were recently found to possess also antitumoral properties, protecting tissues against free radicals, and weak and medium-strength mutagens. An important feature of Rhodiola extracts is their ability to inhibit angiogenesis. Extracts and salidroside stimulated specific and nonspecific immunity both in vivo and in vitro. It seems that they enhance Th1 cytokines without affecting the Th2 profile, thereby ameliorating immunity. To date, however, the molecular mechanisms involved in the different effects remain unclear, and more in vitro and in vivo studies should be conducted.

Rhodiola extracts and salidroside may have potential as coadjuvants of classic chemotherapy, because they modify the tumor microenvironment, increase the antitumoral effect, and lower several adverse effects associated with chemotherapy. The few studies that have been carried out on the antitumoral properties of $R$. rosea extracts suggest that the effect of these compounds may be related to their antioxidant activity, which differs from the typical free radical scavenging mechanism. However, the antitumoral effects of salidroside seem to involve a wide variety of mediators, including cyclin D1, mTOR, and HIF- $1 \alpha$, as well as MAPK, VEGF, STAT3, and JAK2 pathways, among others.

In general, Rhodiola extracts have proven to be more active than the pure compounds salidroside and rosavin. It thus appears that other compounds present in the plants also contribute to the pharmacological activities of the extracts.

\section{Conflict of Interest}

$\nabla$

The authors declare no conflict of interest.

\section{References}

1 Grech-Baran M, Sykłowska-Baranek K, Pietrosiuk A. Biotechnological approaches to enhance salidroside, rosin and its derivatives production in selected Rhodiola spp. in vitro cultures. Phytochem Rev 2015; 14: 657-674

2 GBIF 2010. Biodiversity occurrence data provided by Global Biodiversity Information Facility. Available at http://data.gbif.org. Accessed March 04, 2016

3 European Medicines Agency, Committee on Herbal Medicinal Products. Assessment report on Rhodiola rosea L., rhizoma et radix. EMA/HMPC/ 232100/2011; 27 March 2012

4 Sarwar GR, Qaiser M. Distribution pattern, ecology and endemism of family Crassulaceae in Pakistan and Kashmir. Pak J Bot 2012; 44: 2055-2061

5 Zhengyi W, Raven PH. Flora of China, Vol. 8. Beijing: Science Press and St. Louis: Missouri Botanical Garden Press; 2001: 251-268

6 Radomska-Lesniewska D, Skopinski P, Balan BJ, Bialoszewska A, Józwiak J, Rokicki D, Skopinska-Rózewska E, Borecka A, Hevelke A. Angiomodulatory properties of Rhodiola spp. and other natural antioxidants. Centr Eur J Immunol 2015; 40: 249-262

7 Panossian A, Wikman G, Sarris J. Rosenroot (Rhodiola rosea): traditional use, chemical composition, pharmacology and clinical efficacy. Phytomedicine 2010; 17: 481-493

8 Cuerrier A, Ampong-Nyarko K. Rhodiola rosea. Boca Raton: CRC Press; 2015
9 Panossian A, Hamm R, Kadioglu O, Wilkman G, Efferth T. Synergy antagonism of active constituents of ADAPT-232 on transcriptional level of metabolic regulation of isolated neuroglial cells. Front Neurosci 2013; 7: 1-14

10 Raphael I, Nalawade S, Eagar TN, Forsthuber TG. T cell subsets and their signature cytokines in autoimmune and inflammatory diseases. Cytokine 2015; 74: 5-17

11 Zdanowski $R$, Lewicki S, Skopińska-Różewska E, Buchwald W, Mrozikiewicz M, Stankiewicz W. Alcohol- and water-based extracts obtained from Rhodiola rosea affect differently the number and metabolic activity of circulating granulocytes in Balb/c mice. Ann Agric Environ Med 2014; 21: 120-123

12 Lin SC, Chin LW, Chao PC, Lai YY, Lin LY, Chou MY, Chou MC, Wei JC, Yang CC. In vivo Th1 and Th2 cytokine modulation effects of Rhodiola rosea standardised solution and its major constituent, salidroside. Phytother Res 2011; 25: 1604-1611

13 Hotchkiss RS, Tinsley KW, Karl IE. Role of apoptotic cell death in sepsis. Scand J Infect Dis 2003; 35: 585-592

14 Liu MW, Su MX, Zhang W, Zhang LM, Wang YH, Qian CY. Rhodiola rosea suppresses thymus T-lymphocyte apoptosis by downregulating tumor necrosis factor- $\alpha$-induced protein 8 -like- 2 in septic rats. Int J Mol Med 2015; 36: 386-398

15 Xu X, Tan C, Li P, Zhang S, Pang X, Liu H, Li L, Sun X, Zhang Y, Wu H, Chen $X, G e Q$. Changes of cytokines during a spaceflight analog - a 45day head-down bed rest. PLoS One 2013; 8: e77401

16 Skopińska-Rózewska E, Sokolnicka I, Siwicki AK, Stankiewicz W, Dabrowski MP, Buchwald W, Krajewska-Patan A, Mielcarek S, Mścisz A, Furmanowa M. Dose-dependent in vivo effect of Rhodiola and Echinacea on the mitogen-induced lymphocyte proliferation in mice. Pol J Vet Sci 2011; 14: 265-272

17 Chiang HM, Chien YC, Wu CH, Kuo YH, Wu WC, Pan YY, Su YH, Wen KC. Hydroalcoholic extract of Rhodiola rosea L. (Crassulaceae) and its hydrolysate inhibit melanogenesis in B16F0 cells regulating the CREB/ MITF/tyrosynase pathway. Food Chem Toxicol 2014; 65: 129-139

18 Palumbo DR, Occhiuto F, Spadaro F, Circosta C. Rhodiola rosea extract protects human cortical neurons against glutamate and hydrogen peroxide-induced cell death through reduction in the accumulation of intracellular calcium. Phytother Res 2012; 26: 878-883

19 Liu Z, Li X, Simoneau AR, Jafari M, Zi X. Rhodiola rosea extracts and salidroside decrease the growth of bladder cancer cell lines via inhibition of the mTOR pathway and induction of autophagy. Mol Carcinog 2012; 51: 257-267

20 Cai Z, Li W, Wang H, Yan W, Zhou Y, Wang G, Cui J, Wang F. Antitumor effects of a purified polysaccharide from Rhodiola rosea and its action mechanism. Carbohydr Polym 2012; 90: 296-300

21 Mishra KP, Ganju L, Chanda S, Karan D, Sawhney RC. Aqueous extract of Rhodiola imbricata rhizome stimulates Toll-like receptor 4, granzyme$B$ and Th1 cytokines in vitro. Immunobiology 2009; 214: 27-31

22 Mishra KP, Ganju L, Singh SB. Anti-cellular and immunomodulatory potential of aqueous extract of Rhodiola imbricata rhizome. Immunopharmacol Immunotoxicol 2012; 34: 513-518

23 Diwaker D, Mishra KP, Ganju L, Singh SB. Rhodiola inhibits dengue virus multiplication by inducing innate immune response genes RIG-I, MDA5 and ISG in human monocytes. Arch Virol 2014; 159: 1975-1986

24 Senthilkumar R, Parimelazhagan T, Chaurasia OP, Srivastava RB. Free radical scavenging property and antiproliferative activity of Rhodiola imbricata Edgew extracts in HT-29 human colon cancer cells. Asian Pac J Trop Med 2013; 6: 11-19

25 Chawla R, Jaiswal S, Kumar R, Arora R, Sharma RK. Himalayan bioresource Rhodiola imbricata as a promising radioprotector for nuclear and radiological emergencies. J Pharm Bioallied Sci 2010; 2: 213-219

26 Zhou JT, Li CY, Wang CH, Wang YF, Wang XD, Wang HT, Zhu Y, Jiang MM, Gao XM. Phenolic compounds from the roots of Rhodiola crenulata and their antioxidant and inducing IFN- $\gamma$ production activities. Molecules 2015; 20: 13725-13739

27 Zhu C, Guan F, Wang C, Jin LH. The protective effects of Rhodiola crenulata extracts on Drosophila melanogaster gut immunity induced by bacteria and SDS toxicity. Phytother Res 2014; 28: 1861-1866

28 Bassa LM, Jacobs C, Gregory K, Henchey E, Ser-Dolansky J, Schneider SS. Rhodiola crenulata induces an early estrogenic response and reduced proliferation and tumorsphere formation over time in MCF7 breast cancer cells. Phytomedicine 2016; 23: 87-94

29 Mora MC, Bassa LM, Wong KE, Tirabasi MV, Arenas RB, Schneider SS. Rhodiola crenulata inhibits Wnt/ $\beta$-catenin signaling in glioblastoma. J Surg Res 2015; 197: 247-255 
30 Dudek MC, Wong KE, Bassa LM, Mora MC, Ser-Dolansky J, Henneberry JM, Crisi GM, Arenas RB, Schneider SS. Antineoplastic effects of Rhodiola crenulata treatment on B16-F10 melanoma. Tumour Biol 2015; 36: 9795-9805

31 Li HX, Sze SC, Tong Y, Ng TB. Production of Th1- and Th2-dependent cytokines induced by the Chinese medicine herb, Rhodiola algida, on human peripheral blood monocytes. J Ethnopharmacol 2009; 123: 257-266

32 Loo WTY, Jin LJ, Chow LWC, Cheung MNB, Wang M. Rhodiola algida improves chemotherapy-induced oral mucositis in breast cancer patients. Expert Opin Investig Drugs 2010; 19: S91-S100

33 Qi YJ, Cui S, Lu DX, Yang YZ, Luo Y, Ma L, Ma Y, Wuren T, Chang R, Qi L, Ben BJ, Han J, Ge RL. Effects of the aqueous extract of a Tibetan herb, Rhodiola algida var. tangutica on proliferation and HIF- $1 \alpha$, HIF- $2 \alpha$ expression in MCF-7 cells under hypoxic condition in vitro. Cancer Cell Int 2015; 15: 81

34 Zhang H, Shen WS, Gao CH, Deng LC, Shen D. Protective effects of salidroside on epirubicin-induced early left ventricular regional systolic dysfunction in patients with breast cancer. Drugs R D 2012; 12: 101106

35 Shi TY, Feng SF, Xing JH, Wu YM, Li XQ Zhang N, Tian Z, Liu SB, Zhao MG. Neuroprotective effects of Salidroside and its analogue tyrosol galactoside against focal cerebral ischemia in vivo and $\mathrm{H}_{2} \mathrm{O}_{2}$-induced neurotoxicity in vitro. Neurotox Res 2012; 21: 358-367

36 Zhang JK, Yang L, Meng GL, Yuan Z, Fan J, Li D, Chen JZ, Shi TY, Hu HM, Wei BY, Luo ZJ, Liu J. Protection by salidroside against bone loss via inhibition of oxidative stress and bone-resorbing mediators. PLoS One 2013; 8: e57251

37 Li D, Fu Y, Zhang W, Su G, Liu B, Guo M, Li F, Liang D, Liu Z, Zhang X, Cao Y, Zhang $N$, Yang $Z$. Salidroside attenuates inflammatory responses by suppressing nuclear factor- $\kappa \mathrm{B}$ and mitogen activated protein kinases activation in lipopolysaccharide-induced mastitis in mice. Inflamm Res 2013; 62: 9-15

38 Guan S, He J, Guo W, Wei J, Lu J, Deng X. Adjuvant effects of salidroside from Rhodiola rosea $\mathrm{L}$. on the immune responses to ovalbumin in mice. Immunopharmacol Immunotoxicol 2011; 33: 738-743

$39 \mathrm{Hu}$ X, Lin S, Yu D, Qiu S, Zhang X, Mei R. A preliminary study: the antiproliferation effect of salidroside on different human cancer cell lines. Cell Biol Toxicol 2010; 26: 499-507

40 Liu X, Peng X, Hu Z, Zhao Q, He J, Li J, Zhong X. Effects of over-expression of ANXA10 gene on proliferation and apoptosis of hepatocellular carcinoma cell line HepG2. J Huazhong Univ Sci Technolog Med Sci 2012; 32: 669-674

41 Skopińska-Rózewska E, Malinowski M, Wasiutyński A, Sommer E, Furmanowa M, Mazurkiewicz M, Siwicki AK. The influence of Rhodiola quadrifida $50 \%$ hydro-alcoholic extract and salidroside on tumorinduced angiogenesis in mice. Pol J Vet Sci 2008; 11: 97-104

42 Sun $C$, Wang $Z$, Zheng $Q$ Z Zhang $H$. Salidroside inhibits migration and invasion of human fibrosarcoma HT1080 cells. Phytomedicine 2012; 19 : 355-363

$43 \mathrm{Lu}$ L, Yuan J, Zhang S. Rejuvenating activity of salidroside (SDS): dietary intake of SDS enhances the immune response of aged rats. Age (Dordr) 2013; 35: 637-646

44 Zhao X, Lu Y, Tao Y, Huang Y, Wang D, Hu Y, Liu J, Wu Y, Yu Y, Liu C. Salidroside liposome formulation enhances the activity of dendritic cells and immune responses. Int Immunopharmacol 2013; 17: 1134-1140
45 Hu B, Zou Y, Liu S, Wang J, Zhu J, Li J, Bo L, Deng X. Salidroside attenuates concanavalin A-induced hepatitis via modulating cytokines secretion and lymphocyte migration in mice. Mediators Inflamm 2014; 2014: 314081

46 Chen JJ, Zhang NF, Mao GX, He XB, Zhan YC, Deng HB, Song DQ, Li DD, Li ZR, Si SY, Qiu Q Wang Z. Salidroside stimulates osteoblast differentiation through BMP signaling pathway. Food Chem Toxicol 2013; 62: 499-505

47 Chen $X$, Zhang $Q$, Cheng $Q$ Ding $F$. Protective effect of salidroside against $\mathrm{H}_{2} \mathrm{O}_{2}$-induced cell apoptosis in primary culture of rat hippocampal neurons. Mol Cell Biochem 2009; 332: 85-93

$48 \mathrm{Yu}$ S, Shen Y, Liu J, Ding F. Involvement of ERK1/2 pathway in neuroprotection by salidroside against hydrogen peroxide-induced apoptotic cell death. J Mol Neurosci 2010; 40: 321-331

49 Li X, Ye X, Li X, Sun X, Liang Q Tao L, Kang X, Chen J. Salidroside protects against $\mathrm{MPP}^{+}$-induced apoptosis in PC12 cells by inhibiting the NO pathway. Brain Res 2011; 1382: 9-18

50 Zhang L, Ding W, Sun H, Zhou Q Huang J, Li X, Xie Y, Chen J. Salidroside protects PC12 cells from MPP ${ }^{+}$-induced apoptosis via activation of the PI3K/Akt pathway. Food Chem Toxicol 2012; 50: 2591-2597

51 Xu MC, Gao XF, Ruan C, Ge ZR, Lu JD, Zhang JJ, Zhang Y, Wang L, Shi HM. miR-103 Regulates oxidative stress by targeting the Bcl2/adenovirus E1B 19kDa interacting protein 3 in HUVECs. Oxid Med Cell Longev 2015; 2015: 489647

52 Tan CB, Gao M, Xu WR, Yang XY, Zhu XM, Du GH. Protective effects of salidroside on endothelial cell apoptosis induced by cobalt chloride. Biol Pharm Bull 2009; 32: 1359-1363

$53 \mathrm{Hu}$ X, Lin S, Yu D, Qiu S, Zhang X, Mei R. A preliminary study: the antiproliferation effect of salidroside on different human cancer cell lines. Cell Biol Toxicol 2010; 26: 499-507

54 Zhao G, Shi A, Fan Z, Du Y. Salidroside inhibits the growth of human breast cancer in vitro and in vivo. Oncol Rep 2015; 33: 2553-2560

55 Sun $C$, Wang Z, Zheng $Q$, Zhang $H$. Salidroside inhibits migration and invasion of human fibrosarcoma HT1080 cells. Phytomedicine 2012; 19: 355-363

56 Wang J, Li JZ, Lu AX, Zhang KF, Li BJ. Anticancer effect of salidroside on A459 lung cancer cells through inhibition of oxidative stress and phosphor-p38 expression. Oncol Lett 2014; 7: 1159-1164

57 Zhang Y, Yao Y, Wang H, Guo Y, Zhang H, Chen L. Effects of salidroside on glioma formation and growth inhibition together with improvement of tumor microenvironment. Chin J Cancer Res 2013; 25: 520-526

58 Sun KX, Xia HW, Xia RL. Anticancer effect of salidroside on colon cancer through inhibiting JAK2/STAT3 signaling pathway. Int J Clin Exp Pathol 2015; 8: 615-621

59 Li YR, Cao W, Guo J, Miao S, Ding GR, Li KC, Wang J, Guo GZ. Comparative investigations on the protective effects of rhodioside, ciwujianoside-B and astragaloside IV on radiation injuries of the hematopoietic system in mice. Phytother Res 2011; 25: 644-653

$60 \mathrm{Li}$ X, Sipple J, Pang Q Du W. Salidroside stimulates DNA repair enzyme Parp-1 activity in mouse HSC maintenance. Blood 2012; 119: 41624173

61 Li Q, Zhou XD, Kolosov VP, Perelman JM. Salidroside reduces coldinduced mucin production by inhibiting TRPM8 activation. Int J Mol Med 2013; 32: 637-646

62 Yin $D$, Yao W, Chen S, Hu R, Gao X. Salidroside, the main active compound of Rhodiola plants, inhibits high glucose-induced mesangial cell proliferation. Planta Med 2009; 75: 1191-1195 